\title{
Truth Degrees Theory and Approximate Reasoning in 3-Valued Propositional Pre-Rough Logic
}

\author{
Yingcang Ma, Juanjuan Zhang, and Huan Liu \\ School of Science, Xian Polytechnic University, Xian 710048, China \\ Correspondence should be addressed to Yingcang Ma; mayingcang@126.com
}

Received 5 January 2013; Accepted 6 June 2013

Academic Editor: Francisco Chiclana

Copyright ( 2013 Yingcang Ma et al. This is an open access article distributed under the Creative Commons Attribution License, which permits unrestricted use, distribution, and reproduction in any medium, provided the original work is properly cited.

By means of the function induced by a logical formula $A$, the concept of truth degree of the logical formula $A$ is introduced in the 3 -valued pre-rough logic in this paper. Moreover, similarity degrees among formulas are proposed and a pseudometric is defined on the set of formulas, and hence a possible framework suitable for developing approximate reasoning theory in 3-value logic pre-rough logic is established.

\section{Introduction}

Rough set theory was proposed by Pawlak [1] in the 1980s of the last century to serve as an approximate description of sets which are unknown and incompletely specified. Its applications [2-8] have been found in fields such as data mining, learning, and approximate reasoning. Theoretical work [9-14] has included investigations of the logical, categorytheoretic, topological, and algebraic aspects of rough sets.

It is undoubtedly that set theory and logic systems are strongly coupled in the development of modern logic. Scholars have been trying to build rough logic corresponding to rough set semantics since the birth of rough set theory. The notion of rough logic was initially proposed by Pawlak [9], and this work was followed by Orłowska and Vakarelov in a sequence of papers $[15,16]$. In [10], a formal logic system PRL corresponding to pre-rough algebra was proposed. In [17], Zhang and Zhu proposed a rough logic system RSL whose schematic is rough sets and extensional regular double Stone algebras.

The symbolic and formalism are characteristics in traditional mathematical logic, and the form reasoning of logic was studied mainly through the strict argument. But numerical computation aims to solve various computing problems by means of some mathematical methods and pays close attention to problem solving as well as to error estimation. Hence, mathematical logic and numerical computation are two branches of mathematics miles apart, and it seems that contact between them will not be built.

However, the contact of mathematical logic and numerical calculation is proposed by Wang and Zhou and how put numerical computation into mathematical logic is made a detailed study in his article that Quantitative Logic [18]. The theory of truth degrees of propositions in 2-valued propositional logic was proposed in [19]. In the following, the theory of truth degrees of Lukasiewicz system and other fuzzy logical systems is studied [20-23].

The basic idea of quantitative logic is to provide a graded approach to propositional logic. For example, $p$ is an atomic formula in 2-valued logic; that is, $p$ can choose two values, true and false. That is, $p$ has two valuations true and false, the valuation's sum is 2 , and the number of the valuations which is true is 1 , and then the ratio $1 / 2$ can describe the degree in which the atom formula $p$ is true in all valuations. We can describe each formula $A$ like this. For example, $p \wedge q$ is four kinds of valuations, and the number of the valuations which is true is 1 , so the ratio $1 / 4$ can describe the degree in which the formula $p \wedge q$ is true in all valuations. For many-valued logic, we can like this use the ratio to describe the degree of a proposition which is true in all valuations, and we call the ratio the truth degree of a formula.

In the present paper, according to the method mentioned previously, we introduce a definition of truth degrees on 3 -valued propositional pre-rough logic. We also study the 
theory of truth degree and approximate reasoning theory in 3 -valued pre-rough logic deeply.

The paper is organized as follows. After this introduction, in Section 2 we will introduce the basic content of pre-rough algebra and pre-rough logic in [10]. In Section 3, the theory of truth degree in 3-valued propositional pre-rough logic is built. In Section 4, the similarity degree and pseudodistance between two formulas are defined, and the continuity of operators based on pseudometrics is proven. In Section 5, a type of metric approximate reasoning theory in pre-rough logic based on the proposed pseudometrics is established. The final section offers the conclusion.

\section{Pre-Rough Algebra and Pre-Rough Logic}

Rough set theory begins with information systems (or information tables) and as an abstraction that are proposed. In general, approximation space is a pair $(U, R)$, where $U$ is a nonempty set (the domain of discourse) and $R$ is an equivalence relation on $U$.

Let $(U, R)$ be an approximation space; if $A \subseteq U$, the lower approximation $\underline{R} A$ of $A$ is the union of equivalence classes contained in $A$, while its upper approximation $\bar{R} A$ is the union of equivalence classes intersecting $A$; that is,

$$
\begin{gathered}
\underline{R} A=\cup\{[x] \mid[x] \subseteq A\}=\{x \mid x \in U,[x] \subseteq A\}, \\
\bar{R} A=\cup\{[x] \mid[x] \cap X \neq \phi\}=\{x \mid x \in U,[x] \cap A \neq \phi\},
\end{gathered}
$$

where $[x]$ denotes the equivalence class.

Then we call $\underline{R} A(\bar{R} A)$ rough lower (upper) approximation of $A$. A rough set $A$ is termed definable in $(U, R)$ if and only if $R A=\bar{R} A$.

Definition 1 (see [10]). A structure $\mathscr{P}=(P, \leq, \sqcap, \sqcup, \neg, L, \rightarrow$, $0,1)$ is a pre-rough algebra if and only if

(1) $P=(P, \leq, \sqcap, \sqcup, \neg, L, \rightarrow, 0,1)$ is a bounded distributive lattice with least element 0 and largest element 1 ,

(2) $\neg \neg a=a$,

(3) $\neg(a \sqcup b)=\neg a \sqcap \neg b$,

(4) $L a \leq a$,

(5) $L(a \sqcap b)=L a \sqcap L b$,

(6) $L L a=L a$,

(7) $L 1=1$,

(8) $M L a=L a$,

(9) $\neg L a \sqcup L a=1$,

(10) $L(a \sqcup b)=L a \sqcup L b$,

(11) $L a \leq L b, M a \leq M b$ imply $a \leq b$,

(12) $a \rightarrow b=(\neg L a \sqcup L b) \sqcap(\neg M a \sqcup M b)$.

Example 2. Let $\mathscr{T}=(\{0,1 / 2,1\}, \leq, \Pi, \sqcup, \neg, L, \rightarrow, 0,1)$, where $\leq$ is the usual order on real numbers and $\Pi$ and $\sqcup$ are maximum and minimum, respectively. $\neg 0=1, \neg 1 / 2=1 / 2$, $\neg 1=0, L 0=L(1 / 2)=0$, and $L 1=1$. Then it is a pre-rough algebra and is the smallest nontrivial pre-rough algebra.
The language of pre-rough logic consists of atomic formula $S=\{p, q, r, \ldots\}$, logical symbols $\neg, \Pi$, and $L$, and parentheses.

The formula set of pre-rough logic is generated by the following three rules in finite times:

(i) if $A$ is an atomic formula, then $A$ is a formula;

(ii) if $A$ and $B$ are formulas, then $\neg A, A \sqcap B$, and $L A$ are formulas.

The set of all formulas in pre-rough logic is denoted by $F(S)$. Further connectives are defined as follows, for any wffs $A, B$ of pre-rough logic:

$$
\begin{gathered}
A \sqcup B=\neg A \sqcap \neg B, \\
M A=\neg L \neg A, \\
A \longrightarrow B=(\neg L A \sqcup L B) \sqcap(\neg M A \sqcup M B) .
\end{gathered}
$$

Definition 3 (see [10]). The axioms of pre-rough logic consist of the formulas of the following form:

(1) $A \rightarrow A$,

(2) $\neg \neg A \rightarrow A$,

(3) $A \rightarrow \neg \neg A$,

(4) $A \sqcap B \rightarrow A$,

(5) $A \sqcap B \rightarrow B \sqcap A$,

(6) $A \sqcap(B \sqcup C) \rightarrow(A \sqcap B) \sqcup(A \sqcap C)$,

(7) $(A \sqcap B) \sqcup(A \sqcap C) \rightarrow A \sqcap(B \sqcup C)$,

(8) $L A \rightarrow A$,

(9) $L(A \sqcap B) \rightarrow L A \sqcap L B$,

(10) $L A \sqcap L B \rightarrow L(A \sqcap B)$,

(11) $L A \rightarrow L L A$,

(12) $M L A \rightarrow L A$,

(13) $L(A \sqcup B) \rightarrow L A \sqcup L B$,

(14) $L A \sqcup L B \rightarrow L(A \sqcup B)$.

Rules of inference are as follows

(1) $M P$ rule: $\{A, A \rightarrow B\} \vdash B$,

(2) HS rule: $\{A \rightarrow B, B \rightarrow C\} \vdash A \rightarrow C$,

(3) $\{A\} \vdash B \rightarrow A$,

(4) $\{A \rightarrow B\} \vdash \neg B \rightarrow \neg A$,

(5) $\{A \rightarrow B, A \rightarrow C\} \vdash A \rightarrow B \sqcap C$,

(6) $\{A \rightarrow B, B \rightarrow A, C \rightarrow D, D \rightarrow C\} \vdash(A \rightarrow$ $C) \rightarrow(B \rightarrow D)$,

(7) $\{A \rightarrow B\} \vdash L A \rightarrow L B$,

(8) $\{A\} \vdash L A$,

(9) $\{L A \rightarrow L B, M A \rightarrow M B\} \vdash A \rightarrow B$. 
Definition 4 (see [10]). A valuation $v$ in pre-rough logic is a map from the set of rough formulas $F(S)$ to any pre-rough algebra $\mathscr{P}=(P, \leq, \sqcap, \sqcup, \neg, L, \rightarrow, 0,1)$ satisfying, for all $A, B \in$ $F(S)$,

$$
\begin{gathered}
v(A \sqcap B)=v(A) \sqcap v(B), \\
v(L A)=L(v(A)), \\
v(\neg A)=\neg v(A) .
\end{gathered}
$$

That is, $v$ is $(\neg, \sqcap, L)$ type homomorphism.

Remark 5. With $(\sqcup, \rightarrow, M)$ being defined by $(\neg, \sqcap, L), v$ is also $(\sqcup, \rightarrow, M)$ type homomorphism. That is, $v(A \sqcup B)=$ $v(A) \sqcup v(B), v(A \rightarrow B)=v(A) \rightarrow v(B)$, and $v(M A)=$ $M(v(A))$.

Remark 6. From [10], pre-rough logic is sound and complete relative to the class of all pre-rough algebras. That is, for all $A \in F(S), \Gamma \vdash A$ if and only if $\Gamma \vDash A$, where $\Gamma$ is a theory in pre-rough logic, $\Gamma \vdash A$ means that $A$ is a $\Gamma$-conclusion (i.e., $A$ can be deduced from $A \cup \Gamma$ within finite steps by using reasoning rules of pre-rough logic), and $\Gamma \vDash A$ means that $\Gamma$ entails $A$ (i.e., $A$ is satisfied by $\Gamma$ ).

\section{The Theory of Truth Degree}

Definition 7. Let $T=\{0,1 / 2,1\}$ and define logical operations on $T$ as follows: for all $x, y \in T, \neg 0=1, \neg 1 / 2=1 / 2, \neg 1=$ $0, L 0=L(1 / 2)=0, L 1=1, M 0=0, M(1 / 2)=$ $M 1=1, x \sqcap y=\min (x, y), x \sqcup y=\max (x, y)$, and $x \rightarrow y=\left\{\begin{array}{l}0, x>y \\ 1, x \leq y\end{array}, x, y \in\{0,1 / 2,1\}\right.$. Then $T$ is an algebra of type $(\neg, \sqcap, \sqcup, L, M, \rightarrow)$, which is called 3-valued pre-rough logic system, denoted by $\mathscr{T}$.

A valuation of $v: F(S) \rightarrow \mathscr{T}$ is a homomorphism of type $(\neg, \sqcap, \sqcup, L, M, \rightarrow)$, and $v(A)$ is the valuation of $A$ with respect to $v$. The set consisting of all valuations of $F(S)$ is denoted by $\Omega_{3}$.

Suppose that logic formula $A\left(p_{1}, p_{2}, \ldots, p_{n}\right)$ contains $n$ atomic formulas in $F(S)$, and then $A$ can induce a 3 -value function $f_{A}\left(x_{1}, x_{2}, \ldots, x_{n}\right): L_{3}^{n} \rightarrow L_{3}$, where $f_{A}\left(x_{1}, x_{2}, \ldots\right.$, $\left.x_{n}\right)$ is making $x_{1}, x_{2}, \ldots, x_{n}$ composed together by operators $\neg, \Pi$, and $L$, which is similar to the formula $A\left(p_{1}, p_{2}, \ldots, p_{n}\right)$ that makes $p_{1}, p_{2}, \ldots, p_{n}$ composed together by connective $\neg, \sqcap$, and $L$. For example, a formula $A=p \sqcap(q \rightarrow(r \sqcup \neg p))$ can induce the function $f_{A}\left(x_{1}, x_{2}, x_{3}\right)=x_{1} \sqcap\left(x_{2} \rightarrow\left(x_{3} \sqcup\right.\right.$ $\left.\neg x_{1}\right)$ ), where $x_{1}, x_{2}$, and $x_{3}$ can be any value in $L_{3}$. In this way, for each $v \in \Omega_{3}, v(A)=f_{A}\left(v\left(p_{1}\right), v\left(p_{2}\right), \ldots, v\left(p_{n}\right)\right)$, so for each formula $A$, we can induce a function $f_{A}$ according to $A$, which is called the induced function of $A$. From the definition of tautology, we can get that if $A$ is a tautology if and only if its induced function $f_{A}\left(x_{1}, x_{2}, \ldots, x_{n}\right)=1$ holds for all $\left(x_{1}, x_{2}, \ldots, x_{n}\right) \in L_{3}^{n}$.

Definition 8. let $A\left(p_{1}, p_{2}, \ldots, p_{n}\right) \in F(S)$ contain $n$ atomic formulas in 3-valued pre-rough logic system and let
$f_{A}\left(x_{1}, x_{2}, \ldots, x_{n}\right)$ be the induced function of $A$, and then $\tau(A)$ which is the truth degree of $A$ is defined as

$$
\tau(A)=\frac{1}{3^{n}} \times\left|f_{A}^{-1}(1)\right|
$$

where $\left|f_{A}^{-1}(1)\right|$ is the number of valuations satisfying $f_{A}\left(v\left(p_{1}\right), v\left(p_{2}\right), \ldots, v\left(p_{n}\right)\right)=1$.

Define $\tau(M A)(\tau(L A))$ as the upper (lower) truth degree of $A$, denoted by $\bar{\tau}(A)(\underline{\tau}(A))$.

Remark 9. The definition of truth degree in the paper is given by considering the proportion of the valuation satisfying $v(A)=1$ with respect to all valuations for formula $A$, but in [24], the truth degree of formula $A$ is not only has correlation with the valuation satisfying $v(A)=1$, but also has correlation with the valuation satisfying $v(A)=1 / 2$, so the truth degree's definition in [24] is obtained by considering the proportion of all valuations of formula $A$. We know that in fuzzy reasoning, for convenience and utility, logical OR operator usually uses max operator and logical AND operator usually uses min operator, and like this, the definition in this paper is more simple and more easy to compute; moreover, in the following we can see that many properties hold on this definition.

Remark 10. (i) Let $A=A\left(p_{1}, p_{2}, \ldots, p_{n}\right) \in F(S)$, and for all $v \in \Omega_{3}$, we have $v(M A)=1$ if and only if $v(A)=1$, with solving $\tau(A)$

$$
\bar{\tau}(A)=\frac{1}{3^{n}}\left|f_{M A}^{-1}(1)\right|=\frac{1}{3^{n}}\left(\left|f_{A}^{-1}\left(\frac{1}{2}\right)\right|+\left|f_{A}^{-1}(1)\right|\right) .
$$

(ii) In the same way we can get the following:

$$
\underline{\tau}(A)=\frac{1}{3^{n}}\left|f_{L A}^{-1}(1)\right|=\frac{1}{3^{n}}\left|f_{A}^{-1}(1)\right| .
$$

Remark 11. It is clear that $0 \leq \tau(A)(\bar{\tau}(A), \underline{\tau}(A)) \leq 1$ holds for every $A \in F(S)$. Moreover, logically equivalent formulas have the same truth degree.

Proposition 12. Let $(\bar{\tau}, \underline{\tau}) \tau$ be (upper, lower) truth degree. Then

(i) $0 \leq \underline{\tau}(A)=\tau(A) \leq \bar{\tau}(A) \leq 1$,

(ii) $\bar{\tau}(A)=\tau(M A), \underline{\tau}(A)=\tau(L A)$,

(iii) $\tau(A)=1$ if and only if $A$ is a theorem in pre-rough $\operatorname{logic}$,

$\bar{\tau}(A)=1$ if and only if $M A$ is a theorem in pre-rough $\operatorname{logic}$,

$\underline{\tau}(A)=1$ if and only if $L A$ is a theorem in pre-rough $\overline{l o g i c}$

(iv) $\tau(\neg A)=\underline{\tau}(\neg A)=1-\bar{\tau}(A)$,

(v) if $\vdash M A \rightarrow M B$, then $\bar{\tau}(A) \leq \bar{\tau}(B)$,

if $\vdash L A \rightarrow L B$, then $\underline{\tau}(A) \leq \underline{\tau}(B)$,

if $\vdash A \rightarrow B$, then $\tau(A) \leq \tau(B), \underline{\tau}(A) \leq \underline{\tau}(B)$, and $\bar{\tau}(A) \leq \bar{\tau}(B)$, 


$$
\text { (vi) } \begin{aligned}
\tau(A \sqcup B) & =\tau(A)+\tau(B)-\tau(A \sqcap B), \\
\bar{\tau}(A \sqcup B) & =\bar{\tau}(A)+\bar{\tau}(B)-\bar{\tau}(A \sqcap B), \\
\underline{\tau}(A \sqcup B) & =\underline{\tau}(A)+\underline{\tau}(B)-\underline{\tau}(A \sqcap B) .
\end{aligned}
$$

Theorem 13 (truth degree of $M P$ rules). Let $A, B, C \in F(S)$.

(i) If $\tau(A) \geq \alpha, \tau(A \rightarrow B) \geq \beta$, then $\tau(B) \geq \alpha+\beta-1$.

(ii) If $\bar{\tau}(A) \geq \alpha, \bar{\tau}(A \rightarrow B) \geq \beta$, then $\bar{\tau}(B) \geq \alpha+\beta-1$.

(iii) If $\underline{\tau}(A) \geq \alpha, \underline{\tau}(A \rightarrow B) \geq \beta$, then $\underline{\tau}(B) \geq \alpha+\beta-1$.

Proof. (i) $\tau(A)=\left(1 / 3^{n}\right) \times\left|f_{A}^{-1}(1)\right| \geq \alpha$, and then $\left|f_{A}^{-1}(1)\right| \geq$ $3^{n} \cdot \alpha$.

$\tau(A \rightarrow B)=\left(1 / 3^{n}\right) \times\left|f_{A \rightarrow B}^{-1}(1)\right| \geq \beta$, and then $\left|f_{A \rightarrow B}^{-1}(1)\right| \geq 3^{n} \cdot \beta$.

Let $X=A, Y=A \rightarrow B$.

Because $\left|f_{X \sqcap Y}^{-1}(1)\right|=\left|f_{X}^{-1}(1)\right|+\left|f_{Y}^{-1}(1)\right|-\left|f_{X \sqcup Y}^{-1}(1)\right| \geq$ $3^{n} \cdot \alpha+3^{n} \cdot \beta-3^{n}$.

So we should prove $\left|f_{X \sqcap Y}^{-1}(1)\right| \leq\left|f_{B}^{-1}(1)\right|$, with $v(X \sqcap Y)=$ $v(A \sqcap(A \rightarrow B))=v(A \sqcap(\neg M A \sqcup M B) \sqcap(\neg L A \sqcup L B))$. In order to make $v(X \sqcap Y)=1$ hold, then $v(A \sqcap(\neg M A \sqcup$ $M B) \sqcap(\neg L A \sqcup L B))=1$ should hold. That means, $v(A)=1$, $v(\neg M A \sqcup M B)=1$, and $v(\neg L A \sqcup L B)=1$ should hold. So it must be $v(A)=1$ and $v(B)=1$.

So, if $v(A \sqcap B)=1$, then $v(A)=1$ and $v(B)=1$; from the above analysis we have $v(X \sqcap Y)=1$; that is, $\left|f_{X \sqcap Y}^{-1}(1)\right|=$ $\left|f_{A \sqcap B}^{-1}(1)\right|$.

With $\vdash A \sqcap B \rightarrow B$ being in pre-rough logic, so we have $f_{A \sqcap B}^{-1}(1) \subseteq f_{B}^{-1}(1)$; hence $\left|f_{X \sqcap Y}^{-1}(1)\right| \leq\left|f_{B}^{-1}(1)\right|$, and we get $\left|f_{B}^{-1}(1)\right| \geq 3^{n} \cdot \alpha+3^{n} \cdot \beta-3^{n}$, so $\tau(B)=\left|f_{B}^{-1}(1)\right| / 3^{n} \geq \alpha+\beta-1$ holds. $3^{n} \cdot \alpha$.

(ii) $\bar{\tau}(A)=\left(1 / 3^{n}\right) \times\left|f_{M A}^{-1}(1)\right| \geq \alpha$, and then $\left|f_{M A}^{-1}(1)\right| \geq$ $\bar{\tau}(A \rightarrow B)=\tau(M(A \rightarrow B))=\left(1 / 3^{n}\right) \times\left|f_{M(A \rightarrow B)}^{-1}(1)\right|=$ $\left(1 / 3^{n}\right) \times\left|f_{A \rightarrow B}^{-1}(1)\right| \geq \beta$, and then $\left|f_{A \rightarrow B}^{-1}(1)\right| \geq 3^{n} \cdot \beta$.

Let $X=M A, Y=A \rightarrow B$.

Because $\left|f_{X \sqcap Y}^{-1}(1)\right|=\left|f_{X}^{-1}(1)\right|+\left|f_{Y}^{-1}(1)\right|-\left|f_{X \sqcup Y}^{-1}(1)\right| \geq$ $3^{n} \cdot \alpha+3^{n} \cdot \beta-3^{n}$.

So we should move $\left|f_{X \sqcap Y}^{-1}(1)\right| \leq\left|f_{M B}^{-1}(1)\right|$, with $v(X \sqcap Y)=$ $v(M A \sqcap(A \rightarrow B))=v(M A) \sqcap v(A \rightarrow B)$, in order to make $v(X \sqcap Y)=1$ hold, then $v(M A)=1$ and $v(A \rightarrow B)=1$ should hold. By definition to get $v(M A)=1$ and $v(A) \leq v(B)$, $\left|f_{X \sqcap Y}^{-1}(1)\right|=\mid(v(A)=1 / 2, v(B)=1 / 2),(v(A)=1 / 2, v(B)=$ $1),(v(A)=1, v(B)=1) \mid$, being, $\left|f_{\text {MAпMB }}^{-1}(1)\right|=\mid(v(M A)=$ $1, v(M B)=1)|=|(v(A)=1 / 2, v(B)=1 / 2),(v(A)=$ $1 / 2, v(B)=1),(v(A)=1, v(B)=1 / 2),(v(A)=1, v(B)=1) \mid$.

So $\left|f_{X \sqcap Y}^{-1}(1)\right| \leq\left|f_{M A \sqcap M B}^{-1}(1)\right|$, and $\left|f_{M A \sqcap M B}^{-1}(1)\right| \leq$ $\left|f_{M B}^{-1}(1)\right|$.

Hence, $\left|f_{X \sqcap Y}^{-1}(1)\right| \leq\left|f_{M B}^{-1}(1)\right|$.

We have $\left|f_{M B}^{-1}(1)\right| \geq 3^{n} \cdot \alpha+3^{n} \cdot \beta-3^{n}$.

So $\bar{\tau}(B) \geq \alpha+\beta-1$.

(iii) From (6), we have $\tau(A)=\tau(A), \tau(A \rightarrow B)=$ $\tau(A \rightarrow B)$, and $\underline{\tau}(B)=\tau(\bar{B})$, and then $\underline{\tau}(\bar{B}) \geq \alpha+\beta-1$ holds obviously.
Corollary 14. Let $A, B, C \in F(S)$.

(i) If $\tau(A)=1, \tau(A \rightarrow B)=1$, then $\tau(B)=1$.

(ii) If $\bar{\tau}(A)=1, \bar{\tau}(A \rightarrow B)=1$, then $\bar{\tau}(B)=1$.

(iii) If $\underline{\tau}(A)=1, \underline{\tau}(A \rightarrow B)=1$, then $\underline{\tau}(B)=1$.

Theorem 15 (truth degree of $H S$ rules). Let $A, B, C \in F(S)$.

(i) If $\tau(A \rightarrow B) \geq \alpha, \tau(B \rightarrow C) \geq \beta$, then $\tau(A \rightarrow C) \geq$ $\alpha+\beta-1$.

(ii) If $\bar{\tau}(A \rightarrow B) \geq \alpha, \bar{\tau}(B \rightarrow C) \geq \beta$, then $\bar{\tau}(A \rightarrow C) \geq$ $\alpha+\beta-1$

(iii) If $\underline{\tau}(A \rightarrow B) \geq \alpha, \underline{\tau}(B \rightarrow C) \geq \beta$, then $\underline{\tau}(A \rightarrow C) \geq$ $\alpha+\beta-1$

Proof. (i) $\tau(A \rightarrow B)=\left(1 / 3^{n}\right) \times\left|f_{A \rightarrow B}^{-1}(1)\right| \geq \alpha$, so $\left|f_{A \rightarrow B}^{-1}(1)\right| \geq 3^{n} \cdot \alpha$. $3^{n} \cdot \beta$

$\tau(B \rightarrow C)=\left(1 / 3^{n}\right) \times\left|f_{B \rightarrow C}^{-1}(1)\right| \geq \beta$, so $\left|f_{B \rightarrow C}^{-1}(1)\right| \geq$

$\tau(A \rightarrow C)=\left(1 / 3^{n}\right) \times\left|f_{A \rightarrow C}^{-1}(1)\right|$.

Let $E=f_{A \rightarrow B}^{-1}(1)=\left\{v \in \Omega_{3} \mid v((\neg M A \sqcup M B) \sqcap(\neg L A \sqcup\right.$ $L B))=1\}, F=f_{B \rightarrow C}^{-1}(1)=\left\{v \in \Omega_{3} \mid v((\neg M B \sqcup M C) \sqcap(\neg L B \sqcup\right.$ $L C))=1\}$, and $G=f_{A \rightarrow C}^{-1}(1)=\left\{v \in \Omega_{3} \mid v((\neg M A \sqcup M C) \sqcap\right.$ $(\neg L A \sqcup L C))=1\}$.

Let $E_{i} \subseteq E, F_{i} \subseteq F, G_{i} \subseteq G,(i=1,2), E_{1}=\left\{v \in \Omega_{3} \mid\right.$ $v(\neg M A \sqcup M B)=1\} ; E_{2}=\left\{v \in \Omega_{3} \mid v(\neg L A \sqcup L B)=1\right\}$; $F_{1}=\left\{v \in \Omega_{3} \mid v(\neg M B \sqcup M C)=1\right\} ; F_{2}=\left\{v \in \Omega_{3} \mid v(\neg L B \sqcup\right.$ $L C)=1\} ; G_{1}=\left\{v \in \Omega_{3} \mid v(\neg M A \sqcup M C)=1\right\} ; G_{2}=\{v \in$ $\left.\Omega_{3} \mid v(\neg L A \sqcup L C)=1\right\}$, and then $\tau(A \rightarrow B)=|E| / 3^{n}$, $\tau(B \rightarrow C)=|F| / 3^{n}, \tau(A \rightarrow C)=|G| / 3^{n}$. So we should prove $|E|+|F| \leq 3^{n}+|G|$, with $|E|+|F|=|E \cup F|+|E \cap F|$.

We first prove $E \cap F \subseteq G$.

Let $x \in E \cap F$, then $x \in E_{i}, F_{i}(i=1,2)$, from the structure of $E_{i}, F_{i}$ we prove $E \cap F \leq G$ in the following two cases.

Case 1. Let $x \in\left\{v \in \Omega_{3} \mid v(L B)=1\right\}$, and then we can get $x \in\left\{v \in \Omega_{3} \mid v(M B)=1\right\}$, with $x \in F_{1}$, so $x \in\left\{v \in \Omega_{3} \mid\right.$ $v(M C)=1\}$, that is to say, $x \in G_{1}$; with $x \in F_{2}, x \in\left\{v \in \Omega_{3}\right.$ | $v(L C)=1\}$, that is, $x \in G_{2}$. Hence $x \in G_{1} \cap G_{2}=G$.

Case 2. Let $x \notin\left\{v \in \Omega_{3} \mid v(L B)=1\right\}$, in this case we prove $E \cap F \leq G$ in the following two cases.

(1) If $x \in\left\{v \in \Omega_{3} \mid v(M B)=1\right\}$, with $x \in F_{1}$, so $x \in$ $\left\{v \in \Omega_{3} \mid v(M C)=1\right\}$, that is, $x \in G_{1}$; with $x \in E_{2}$, $x \in\left\{v \in \Omega_{3} \mid \neg v(L A)=1\right\}$, that is, $x \in G_{2}$. Hence $x \in G_{1} \cap G_{2}=G$.

(2) If $x \notin\left\{v \in \Omega_{3} \mid v(M B)=1\right\}$, with $x \in E_{1}$, so $x \in$ $\left\{v \in \Omega_{3} \mid v(\neg M A)=1\right\}$, that is, $x \in G_{1}$; with $x \in E_{2}$, $x \in G_{2}$. Hence $x \in G_{1} \cap G_{2}=G$.

So we have $E \cap F \subseteq G \Rightarrow|E \cap F| \leq|G|$.

With $|E|+|F|=|E \cup F|+|E \cap F|$ and $|E \cup F| \leq 3^{n}$, we can get

$$
|E|+|F|=|E \cup F|+|E \cap F| \leq 3^{n}+|G| .
$$

That is, $\left|f_{A \rightarrow B}^{-1}(1)\right|+\left|f_{B \rightarrow C}^{-1}(1)\right| \leq 3^{n}+\left|f_{A \rightarrow C}^{-1}(1)\right|$.

So $\tau(A \rightarrow B)+\tau(B \rightarrow C) \leq 1+\tau(A \rightarrow C)$, and hence $\tau(A \rightarrow C) \geq \alpha+\beta-1$ holds. 
(ii) From the definition, with $v(M(A \rightarrow B))=v(A \rightarrow$ $B), v(M(B \rightarrow C))=v(B \rightarrow C), v(M(A \rightarrow C))=v(A \rightarrow$ $C)$, so $\bar{\tau}(A \rightarrow C) \geq \alpha+\beta-1$ holds obviously.

(iii) From the definition, with $v(L(A \rightarrow B))=v(A \rightarrow$ $B), v(L(B \rightarrow C))=v(B \rightarrow C), v(L(A \rightarrow C))=v(A \rightarrow C)$, so $\underline{\tau}(A \rightarrow C) \geq \alpha+\beta-1$ holds obviously.

Corollary 16. Let $A, B, C \in F(S)$. If $\tau(A \rightarrow B)=1, \tau(B \rightarrow$ $C)=1$, then $\tau(A \rightarrow C)=1$.

\section{Similarity Degree and a Pseudometric among Formulas}

Definition 17. Suppose that $A, B \in F(S)$. Let

$$
\begin{gathered}
\xi(A, B)=\tau((A \longrightarrow B) \sqcap(B \longrightarrow A)), \\
\bar{\xi}(A, B)=\tau((M A \longrightarrow M B) \sqcap(M B \longrightarrow M A)), \\
\underline{\xi}(A, B)=\tau((L A \longrightarrow L B) \sqcap(L B \longrightarrow L A)) .
\end{gathered}
$$

Then one calls $(\bar{\xi}(A, B), \xi(A, B)) \xi(A, B)$ the (upper, lower) similarity degree between $A$ and $B$.

Example 18. Let $A=p, B=q$, where $p, q$ are different atomic formulas, and calculate $\xi(A, B), \bar{\xi}(A, B), \underline{\xi}(A, B)$.

Solution 1. Consider

$$
\begin{aligned}
\xi(A, B) & =\xi(p, q)=\tau((p \longrightarrow q) \sqcap(p \longrightarrow q)) \\
& =\frac{1}{3^{2}} \cdot\left|f_{(p \rightarrow q) \sqcap(q \rightarrow p)}^{-1}(1)\right| .
\end{aligned}
$$

With $f_{(p \rightarrow q) \sqcap(q \rightarrow p)}^{-1}(1)=\{(0,0),(1 / 2,1 / 2),(1,1)\}$, so $\xi(A$, B) $=3 / 3^{2}=1 / 3$. Consider

$$
\begin{aligned}
\bar{\xi}(A, B) & =\bar{\xi}(p, q)=\tau((M p \rightarrow M q) \sqcap(M p \longrightarrow M q)) \\
& =\frac{1}{3^{2}} \cdot\left|f_{(M p \rightarrow M q) \sqcap(M q \rightarrow M p)}^{-1}(1)\right| .
\end{aligned}
$$

With $f_{(M p \rightarrow M q) \sqcap(M q \rightarrow M p)}^{-1}(1)=\{(0,0),(1 / 2,1 / 2),(1 / 2$, $1),(1,1 / 2),(1,1)\}$, so $\bar{\xi}(A, B)=5 / 3^{2}=5 / 9$.

Similarly, we can obtain $\underline{\xi}(A, B)=5 / 9$.

Proposition 19. Suppose that $A, B, C \in F(S)$, and then

(i) $\bar{\xi}(A, B)=\xi(M A, M B), \xi(A, B)=\xi(L A, L B)$,

(ii) $\xi(A, B)=1$ if and only if $\vdash A \leftrightarrow B$,

$$
\begin{aligned}
& \bar{\xi}(A, B)=1 \text { if and only if } \vdash M A \leftrightarrow M B, \\
& \underline{\xi}(A, B)=1 \text { if and only if } \vdash L A \leftrightarrow L B,
\end{aligned}
$$

(iii) $\xi(A, B)+\xi(B, C) \leq \xi(A, C)+1$,

$$
\begin{aligned}
& \bar{\xi}(A, B)+\bar{\xi}(B, C) \leq \bar{\xi}(A, C)+1, \\
& \underline{\xi}(A, B)+\underline{\xi}(B, C) \leq \underline{\xi}(A, C)+1,
\end{aligned}
$$

(iv) $\bar{\xi}(A, B)=0$ if and only if $\vdash M A \leftrightarrow \neg M B$, $\underline{\xi}(A, B)=0$ if and only if $\vdash L A \leftrightarrow \neg L B$,

(v) $\xi(A, B)=\xi(B, A), \bar{\xi}(A, B)=\bar{\xi}(B, A), \underline{\xi}(A, B)=\underline{\xi}(B$, A).

Proof. The proof of (i), (ii), and (v) is obvious; we only give the proof of (iii) and (iv).

(iii) In general, assume that $A, B$, and $C$ have the same atomic formulas $p_{1}, \ldots, p_{n}$.

Let $E=f_{(A \rightarrow B) \sqcap(B \rightarrow A)}^{-1}(1), F=f_{(B \rightarrow C) \sqcap(C \rightarrow B)}^{-1}(1)$, and $G=f_{(A \rightarrow C) \sqcap(C \rightarrow A)}^{-1}(1)$.

Then $\xi(A, B)+\xi(B, C)=|E| / 3^{n}+|F| / 3^{n}, \xi(A, B)+1=$ $|G| / 3^{n}+1$.

And with $E \cap F \subseteq G$, we have $|E|+|F|=|E \cup F|+|E \cap F| \leq$ $3^{n}+|G| ;$ that is,

$$
\begin{aligned}
& \left|f_{(A \rightarrow B) \sqcap(B \rightarrow A)}^{-1}(1)\right|+\left|f_{(B \rightarrow C) \sqcap(C \rightarrow B)}^{-1}(1)\right| \\
& \quad \leq 3^{n}+\left|f_{(A \rightarrow C) \sqcap(C \rightarrow A)}^{-1}(1)\right| .
\end{aligned}
$$

Hence, $\xi(A, B)+\xi(B, C) \leq \xi(A, C)+1$.

The proof of the other inequalities can be obtained in a similar way.

(iv) From (9), we can get $\bar{\xi}(A, B)=0 \Leftrightarrow \tau((M A \rightarrow$ $M B) \sqcap(M B \rightarrow M A))=0$,

$$
\begin{aligned}
& \Leftrightarrow \text { for all } v \in \Omega_{3}, \quad v((M A \rightarrow M B) \sqcap(M B \rightarrow M A))= \\
& 0, \\
& \Leftrightarrow \text { for all } v \in \Omega_{3}, \quad v((M A \rightarrow M B))=0 \text { or } v((M B \rightarrow \\
& M A))=0, \\
& \Leftrightarrow \text { for all } v \in \Omega_{3}, \quad v(M A)=\neg v(M B), \\
& \Leftrightarrow \text { for all } v \in \Omega_{3}, \quad v(M A)=v(\neg M B), \\
& \Leftrightarrow \vdash M A \leftrightarrow \neg M B .
\end{aligned}
$$

In the same way, we can get $\xi(A, B)=0$ if and only if $\vdash L A \leftrightarrow \neg L B$

Definition 20. Suppose that $A, B \in F(S)$, and $A$ and $B$ are said to be similar if $\xi(A, B)=1$.

Definition 20 gives a similarity relation in $F(S)$, and we can easily get the following corollary from Proposition 19.

Corollary 21. The similarity relation among formulas is an equivalent relation.

Proposition 22. Suppose that $A, B, A^{\prime}, B^{\prime} \in F(S)$ and $\xi(A$, $\left.A^{\prime}\right) \geq \alpha, \xi\left(B, B^{\prime}\right) \geq \beta$, and then

$$
\xi\left(A \longrightarrow B, A^{\prime} \longrightarrow B^{\prime}\right) \geq \alpha+\beta-1 .
$$

Proof. Let $C=\left(A^{\prime} \rightarrow A\right) \rightarrow\left((A \rightarrow B) \rightarrow\left(A^{\prime} \rightarrow B\right)\right)$, which is a tautology in 3 -valued pre-rough logic and hence $\tau(C)=1$. Moreover, $\tau\left(A^{\prime} \rightarrow A\right) \geq \xi\left(A, A^{\prime}\right) \geq \alpha$, and hence it follows from Theorem 13(i) that $\tau\left((A \rightarrow B) \rightarrow\left(A^{\prime} \rightarrow\right.\right.$ $B)) \geq \alpha+1-1=\alpha$. Similarly $\tau\left(\left(A^{\prime} \rightarrow B\right) \rightarrow(A \rightarrow B)\right) \geq \alpha$. It follows that $\xi\left(A \rightarrow B, A^{\prime} \rightarrow B\right) \geq \alpha$. On the other hand, it 
can be proved from $\xi\left(B, B^{\prime}\right) \geq \beta$ that $\xi\left(A^{\prime} \rightarrow B, A^{\prime} \rightarrow B^{\prime}\right) \geq$ $\beta$. From Proposition 19(iii) $\xi\left(A \rightarrow B, A^{\prime} \rightarrow B^{\prime}\right) \geq \alpha+\beta-1$ holds.

Definition 23. Define three nonnegative functions $\rho, \bar{\rho}, \rho$ : $F(S) \times F(S) \rightarrow[0,1]$ as follows: for all $A, B \in F(S)$,

$$
\begin{aligned}
& \rho(A, B)=1-\xi(A, B), \\
& \bar{\rho}(A, B)=1-\bar{\xi}(A, B), \\
& \underline{\rho}(A, B)=1-\underline{\xi}(A, B) .
\end{aligned}
$$

Then $\rho,(\bar{\rho}, \rho)$ is called the pseudometrics on the set of rough formulas in pre-rough logic.

Proposition 24 is obviously and which can state $\rho, \bar{\rho}, \rho$ are indeed the pseudometrics on the set of rough formulas in prerough logic.

Proposition 24. Let $\rho, \bar{\rho}$, and $\rho$ be the three nonnegative functions defined in the above definition, and then for all $A, B \in$ $F(S)$

$$
\begin{aligned}
(1) \rho(A, A) & =\bar{\rho}(A, A)=\underline{\rho}(A, A)=0 . \\
(2) \rho(A, B) & =\rho(B, A), \\
\bar{\rho}(A, B) & =\bar{\rho}(B, A), \\
\underline{\rho}(A, B) & =\underline{\rho}(B, A) . \\
\text { (3) } \rho(A, C) & \leq \rho(A, B)+\rho(B, C), \\
\bar{\rho}(A, C) & \leq \bar{\rho}(A, B)+\bar{\rho}(B, C), \\
\underline{\rho}(A, C) & \leq \underline{\rho}(A, B)+\underline{\rho}(B, C) .
\end{aligned}
$$

Theorem 25. The operators $\neg, L$, and $\Pi$ are continuous on $(F(S), \rho)$.

Proof. Suppose that $A, B \in F(S), \varepsilon>0$, and $\rho(A, B)<\varepsilon$, and then $\rho(\neg A, \neg B)=1-\xi(\neg A, \neg B)=1-\tau((\neg A \rightarrow \neg B) \sqcap(\neg B \rightarrow$ $\neg A))=1-\tau((B \rightarrow A) \sqcap(A \rightarrow B))=1-\xi(A, B)=\rho(A, B)<$ $\varepsilon$. Hence $\neg: F(S) \rightarrow F(S)$ is continuous.

Similarly, suppose that $A, B \in F(S), \varepsilon>0$, and $\rho(A, B)<$ $\varepsilon$, then $\rho(L A, L B)=1-\xi(L A, L B)=1-\tau((L A \rightarrow L B) \sqcap$ $(L B \rightarrow L A)) \leq 1-\tau((A \rightarrow B) \sqcap(B \rightarrow A))=1-\xi(A, B)=$ $\rho(A, B) \leq \varepsilon$. Hence $L: F(S) \rightarrow F(S)$ is continuous.

Suppose that $A, B, A^{\prime}, B^{\prime} \in F(S)$ and $\rho\left(A, A^{\prime}\right) \leq \varepsilon / 2$, $\rho\left(B, B^{\prime}\right) \leq \varepsilon / 2$, and then $\rho\left(A \sqcap B, A^{\prime} \sqcap B^{\prime}\right)=1-\xi\left(A \sqcap B, A^{\prime} \sqcap B^{\prime}\right)$, Being $\xi\left(A \sqcap B, A^{\prime} \sqcap B\right)+\xi\left(B \sqcap A^{\prime}, B^{\prime} \sqcap A^{\prime}\right)=\tau(((A \sqcap B) \rightarrow$ $\left.\left.\left(A^{\prime} \sqcap B\right)\right) \sqcap\left(\left(A^{\prime} \sqcap B\right) \rightarrow(A \sqcap B)\right)\right)+\tau\left(\left(\left(B \sqcap A^{\prime}\right) \rightarrow\right.\right.$ $\left.\left.\left(B^{\prime} \sqcap A^{\prime}\right)\right) \sqcap\left(\left(B^{\prime} \sqcap A^{\prime}\right) \rightarrow\left(B \sqcap A^{\prime}\right)\right)\right) \geq \tau\left(\left(A \rightarrow A^{\prime}\right) \sqcap\left(A^{\prime} \rightarrow\right.\right.$ $A))+\tau\left(\left(B \rightarrow B^{\prime}\right) \sqcap\left(B^{\prime} \rightarrow B\right)\right)-1 \geq 1-\varepsilon / 2+1-\varepsilon / 2-1=1-\varepsilon$, So $\xi\left(A \sqcap B, A^{\prime} \sqcap B^{\prime}\right) \leq 1-(1-\varepsilon)=\varepsilon$. Hence $\rho\left(A \sqcap B, A^{\prime} \sqcap B^{\prime}\right) \leq \varepsilon$ and $\Pi$ is continuous.

Remark 26. Since $\rightarrow$,,$M$ are abbreviations of operators $\neg, \sqcap, L$, so these operators are also continuous on $(F(S), \rho)$.

\section{Approximate Reasoning in 3-Valued Propositional Pre-Rough Logic}

Definition 27. Let $\Gamma \subset F(S)$, for all $A \in F(S), \varepsilon>0$, if $\rho(A, D(\Gamma))=\inf \{\rho(A, B): B \in D(\Gamma)\}<\varepsilon, \bar{\rho}(A, D(\Gamma))=$ $\inf \{\bar{\rho}(A, B): B \in D(\Gamma)\}<\varepsilon, \rho(A, D(\Gamma))=\inf \{\rho(A, B): B \epsilon$ $D(\Gamma)\}<\varepsilon$. Then we call $A$ an approximate rough (upper, lower) consequence of $\Gamma$ with I-type error less than $\varepsilon$, respectively. Denoted by $A \in D_{\varepsilon}^{1}(\Gamma), A \in \overline{D_{\varepsilon}^{1}}(\Gamma)$, and $A \in D_{\varepsilon}^{1}(\Gamma)$ each other.

Definition 28. Let $\Gamma \subset F(S)$, for all $A \in F(S), \varepsilon>0$, if $1-$ $\sup \{\tau(B \rightarrow A): B \in D(\Gamma)\}<\varepsilon, 1-\sup \{\tau(M B \rightarrow M A):$ $B \in D(\Gamma)\}<\varepsilon, 1-\sup \{\tau(L B \rightarrow L A): B \in D(\Gamma)\}<\varepsilon$. Then we call $A$ an approximate rough (upper, lower) consequence of $\Gamma$ with II-type error less than $\varepsilon$, respectively. Denoted by $A \in D_{\varepsilon}^{2}(\Gamma), A \in \overline{D_{\varepsilon}^{2}}(\Gamma)$, and $A \in \underline{D_{\varepsilon}^{2}}(\Gamma)$ each other.

Definition 29. Let $\Gamma \subset F(S)$, for all $A \in F(S), \varepsilon>$ 0 , if $\inf \{H(D(\Gamma), D(\Sigma)) \quad \mid \Sigma \subset F(S), \Sigma \vdash A\}<$ $\varepsilon, \inf \{\bar{H}(D(\Gamma), D(\Sigma)) \quad \mid \quad \subset \quad F(S), \Sigma \quad \vdash \quad A\}<\varepsilon$, $\inf \{\underline{H}(D(\Gamma), D(\Sigma)) \mid \Sigma \subset F(S), \Sigma \vdash A\}<\varepsilon$. Then we call $A$ an approximate rough (upper, lower) consequence of $\Gamma$ with IIItype error less than $\varepsilon$, respectively. Denoted by $A \in D_{\varepsilon}^{3}(\Gamma)$, $A \in \overline{D_{\varepsilon}^{3}}(\Gamma)$, and $A \in D_{\varepsilon}^{3}(\Gamma)$ each other, and $H$ is Hausdorff distance.

Where $(X, \rho)$ is a metric space, $U$ and $V$ are nonempty subsets on $X$.

Let $\rho(x, V)=\inf \{\rho(x, y): y \in V\} ; \bar{\rho}(x, V)=\inf \{\bar{\rho}(x$, $y): y \in V\} ; \rho(x, V)=\inf \{\rho(x, y): y \in V\}, H^{*}(U, V)=$ $\sup \{\rho(x, V): x \in U\} ; \overline{H^{*}}(U, \bar{V})=\sup \{\bar{\rho}(x, V): x \in U\}$,

$$
\begin{aligned}
& \underline{H^{*}}(U, V)=\sup \{\underline{\rho}(x, V): x \in U\}, \\
& H(U, V)=\max \left(H^{*}(U, V), H^{*}(V, U)\right), \\
& \bar{H}(U, V)=\max \left(\overline{H^{*}}(U, V), \overline{H^{*}}(V, U)\right), \\
& \underline{H}(U, V)=\max \left(\underline{H^{*}}(U, V), \underline{H^{*}}(V, U)\right) .
\end{aligned}
$$

Theorem 30. Let $\Gamma \subset F(S)$, for all $A \in F(S), \varepsilon>0$, and then

(i) $A \in D_{\varepsilon}^{1}(\Gamma)$ if and only if $A \in D_{\varepsilon}^{2}(\Gamma)$,

(ii) $A \in \overline{D_{\varepsilon}^{1}}(\Gamma)$ if and only if $A \in \overline{D_{\varepsilon}^{2}}(\Gamma)$,

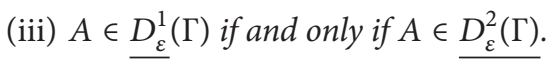

Proof. (i) Let $A \in D_{\varepsilon}^{1}(\Gamma)$, and then we can get $\rho(A, D(\Gamma))=$ $\inf \{\rho(A, B): B \in D(\Gamma)\}<\varepsilon$, for $\rho(A, D(\Gamma))=\inf \{\rho(A, B)$ : $B \in D(\Gamma)\} \leq \inf \{\rho(A, A \sqcup B): B \in D(\Gamma)\}=\inf \{1-\xi(A, A \sqcup B):$ $B \in D(\Gamma)\}=\inf \{1-\tau((A \rightarrow A \sqcup B) \sqcap(A \sqcup B \rightarrow A))\}=$ $\inf \{1-\tau(B \rightarrow A)\}=1-\sup \{\tau(B \rightarrow A)\}<\varepsilon$, so $A \in D_{\varepsilon}^{2}(\Gamma)$.

Let $A \in D_{\varepsilon}^{2}(\Gamma)$, and then we can get $1-\sup \{\tau(B \rightarrow A)$ : $B \in D(\Gamma)\}<\varepsilon$, namely $\sup \{\tau(B \rightarrow A): B \in D(\Gamma)\}>1-\varepsilon$.

For $\sup \{\tau(B \rightarrow A): B \in D(\Gamma)\} \geq \sup \{\tau((B \rightarrow A) \sqcap$ $(A \rightarrow B)): B \in D(\Gamma)\}=\sup \{\xi(B \rightarrow A): B \in D(\Gamma)\}=$ $1-\inf \{1-\xi(B \rightarrow A): B \in D(\Gamma)\}=1-\inf \{\rho(A, B): B \epsilon$ $D(\Gamma)\}=1-\rho(A, D(\Gamma))>1-\varepsilon$, hence $\rho(A, D(\Gamma))<\varepsilon$, so $A \in D_{\varepsilon}^{1}(\Gamma)$. 
In conclusion, $A \in D_{\varepsilon}^{1}(\Gamma)$ if and only if $A \in D_{\varepsilon}^{2}(\Gamma)$ is established. (i).

The proof of (ii) and (iii) can be in a similar way as that of

Theorem 31. Let $\Gamma \subset F(S)$, for all $A \in F(S), \varepsilon>0$, and then

(i) if $A \in D_{\varepsilon}^{3}(\Gamma)$, then $A \in D_{\varepsilon}^{1}(\Gamma)$;

(ii) if $A \in \overline{D_{\varepsilon}^{3}}(\Gamma)$, then $A \in \overline{D_{\varepsilon}^{1}}(\Gamma)$;

(iii) if $A \in \underline{D_{\varepsilon}^{3}}(\Gamma)$, then $A \in \underline{D_{\varepsilon}^{1}(\Gamma) \text {. }}$

Proof. (i) Let $A \in D_{\varepsilon}^{3}(\Gamma)$, and we can get $\inf \{H(D(\Gamma), D(\Sigma)) \mid$ $\Sigma \subset F(S), \Sigma \vdash A\}<\varepsilon$, so there exists $\Sigma \subset F(S)$ makes $\Sigma \vdash A$ and $H(D(\Gamma), D(\Sigma))<\varepsilon$ hold. At the same time $A \in D(\Sigma)$, $\rho(A, D(\Gamma)) \leq H(D(\Gamma), D(\Sigma))<\varepsilon$.

Hence we can get $A \in D_{\varepsilon}^{1}(\Gamma)$. (i).

The proof of (ii) and (iii) can be in a similar way as that of

\section{Conclusion}

Through the basic method of quantitative logic, the theory of truth degree in 3-valued pre-rough logic is studied in this paper. Moreover, similarity degrees among formulas are proposed and a pseudometric is defined therefrom on the set of formulas, and hence a possible framework suitable for developing approximate reasoning theory in 3-valued prerough logic is established.

It is worthy to notice that the results obtained in the present paper are based on the assumption that the probabilities involved are evenly distributed, while it may happen in real life that some propositions are considered more important than others and hence should be endowed with higher probabilities. Therefore, how to combine the method of probability logic is an attractive research topic, and we can find paper $[21,22]$ have give a possible way combine fuzzy logic and probability logic.

\section{Acknowledgments}

This work is supported by the Scientific Research Program Funded by Shaanxi Provincial Education Department (Program no. 12JK0878) and Doctor Scientific Research Foundation Program of Xian Polytechnic University.

\section{References}

[1] Z. Pawlak, "Rough sets," International Journal of Computer and Information Sciences, vol. 11, no. 5, pp. 341-356, 1982.

[2] Md. A. Khan and M. Banerjee, "Formal reasoning with rough sets in multiple-source approximation systems," International Journal of Approximate Reasoning, vol. 49, no. 2, pp. 466-477, 2008.

[3] Y. Leung, M. M. Fischer, W.-Z. Wu, and J.-S. Mi, "A rough set approach for the discovery of classification rules in intervalvalued information systems," International Journal of Approximate Reasoning, vol. 47, no. 2, pp. 233-246, 2008.
[4] A. Mieszkowicz-Rolka and L. Rolka, "Fuzzy rough approximations of process data," International Journal of Approximate Reasoning, vol. 49, no. 2, pp. 301-315, 2008.

[5] Z. Xu and R. R. Yager, "Dynamic intuitionistic fuzzy multiattribute decison making," International Journal of Approximate Reasoning, vol. 48, no. 1, pp. 246-262, 2008.

[6] Y. Yao, "Probabilistic rough set approximations," International Journal of Approximate Reasoning, vol. 49, no. 2, pp. 255-271, 2008.

[7] Y. Leung, J.-M. Ma, W.-X. Zhang, and T.-J. Li, "Dependencespace-based attribute reductions in inconsistent decision information systems," International Journal of Approximate Reasoning, vol. 49, no. 3, pp. 623-630, 2008.

[8] Y. Yao, "The superiority of three-way decisions in probabilistic rough set models," Information Sciences, vol. 181, no. 6, pp. 10801096, 2011.

[9] Z. Pawlak, "Rough logic," Bulletin of the Polish Academy of Sciences, vol. 35, no. 5-6, pp. 253-258, 1987.

[10] M. Banerjee and M. K. Chakraborty, "Rough sets through algebraic logic," Fundamenta Informaticae, vol. 28, no. 3-4, pp. 211221, 1996.

[11] I. Düntsch, "A logic for rough sets," Theoretical Computer Science, vol. 179, no. 1-2, pp. 427-436, 1997.

[12] M. K. Chakraborty and M. Banerjee, "Rough consequence," Polish Academy of Sciences, vol. 41, no. 4, pp. 299-304, 1993.

[13] M. Banerjee, "Logic for rough truth," Fundamenta Informaticae, vol. 71, no. 2-3, pp. 139-151, 2006.

[14] M. Banerjee and Md. A. Khan, "Propositional logics from rough set theory," in Transactions on Rough Sets. VI, vol. 4374 of Lecture Notes in Computer Science, pp. 1-25, Springer, Berlin, Germany, 2007.

[15] E. Orłowska, "Kripke semantics for knowledge representation logics," Studia Logica, vol. 49, no. 2, pp. 255-272, 1990.

[16] D. Vakarelov, "A modal logic for similarity relations in Pawlak knowledge representation systems," Fundamenta Informaticae, vol. 15, no. 1, pp. 61-79, 1991.

[17] X.-H. Zhang and F. Zhu, "Rough logic system RSL and fuzzy logic system Luk," Journal of the University of Electronic Science and Technology of China, vol. 40, no. 2, pp. 296-302, 2011.

[18] G. Wang and H. Zhou, "Quantitative logic," Information Sciences, vol. 179, no. 3, pp. 226-247, 2009.

[19] G. Wang, L. Fu, and J. Song, "Theory of truth degrees of propositions in two-valued logic," Science in China A, vol. 45, no. 9, pp. 1106-1116, 2002.

[20] J. Li and G. Wang, "Theory of truth degrees of propositions in the logic system $\mathrm{L}_{n}^{*}$, Science in China F, vol. 49, no. 4, pp. 471$483,2006$.

[21] X. J. Hui, "Generalization of the fundamental theorem of probability logic in multi-valued propositional logic," Acta Mathematicae Applicatae Sinica, vol. 34, no. 2, pp. 217-228, 2011.

[22] X. J. Hui, "Randomized divergence degree of the theories in the $R_{0} 3$-valued propositional logic system," Acta Mathematicae Applicatae Sinica, vol. 33, no. 1, pp. 142-149, 2010.

[23] G. J. Wang and H. X. Shi, "Quantitative research of generalized tautologies in $n$-valued propositional logic $\mathrm{L}_{n}^{*}$," Journal of Shaanxi Normal University, vol. 37, no. 2, pp. 1-5, 2009.

[24] Y. She, X. He, and G. Wang, "Rough truth degrees of formulas and approximate reasoning in rough logic," Fundamenta Informaticae, vol. 107, no. 1, pp. 67-83, 2011. 


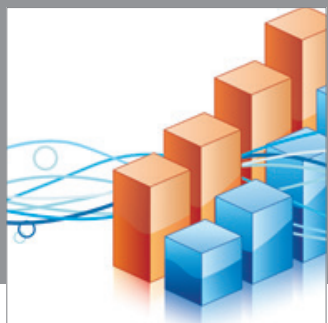

Advances in

Operations Research

mansans

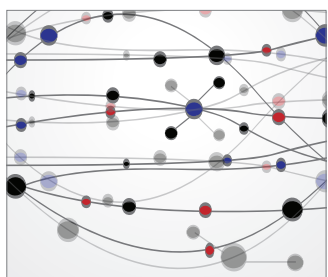

The Scientific World Journal
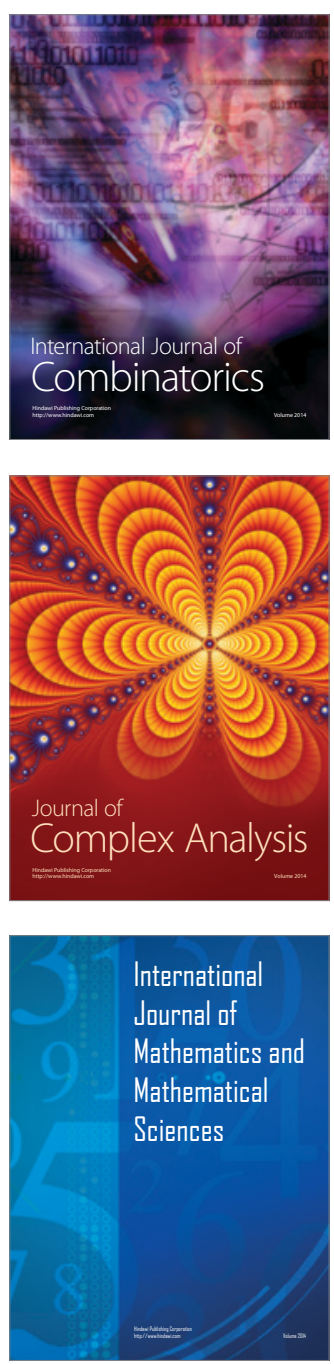
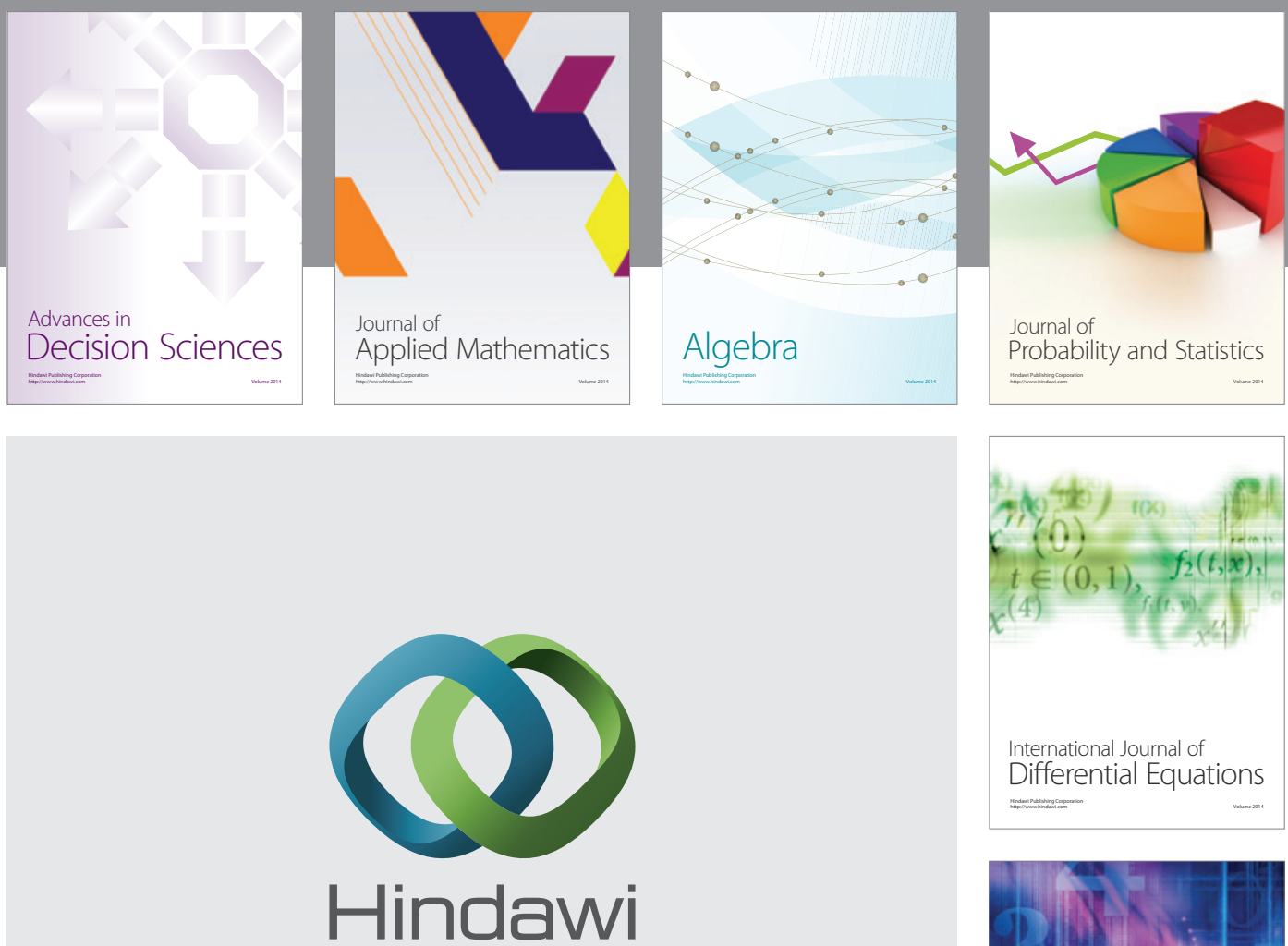

Submit your manuscripts at http://www.hindawi.com
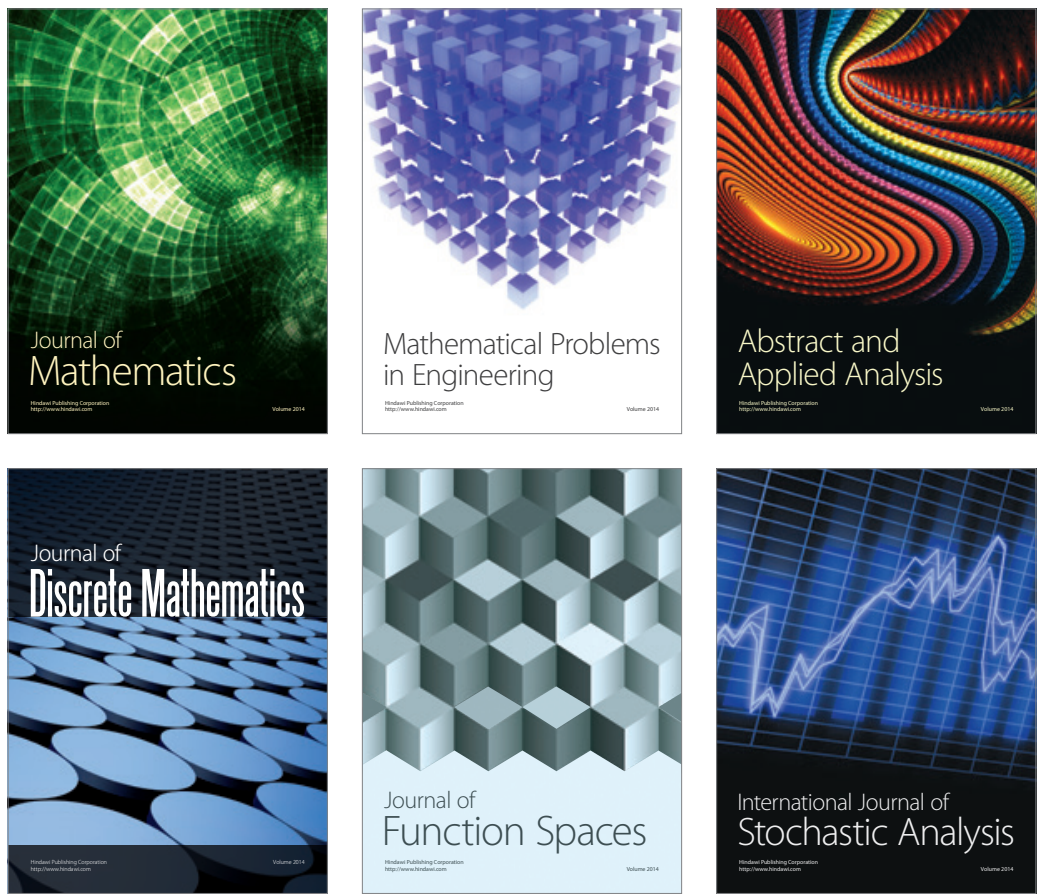

Journal of

Function Spaces

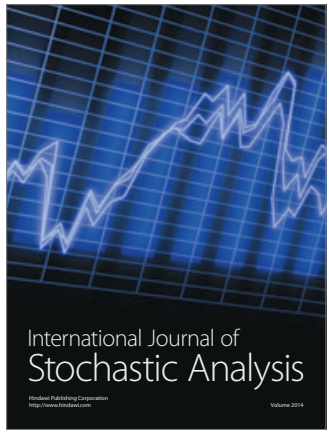

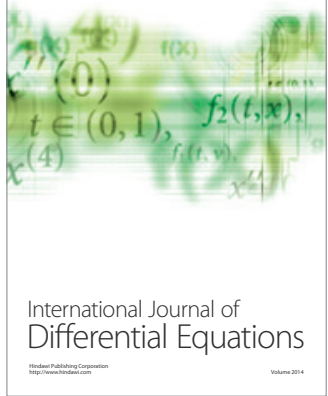
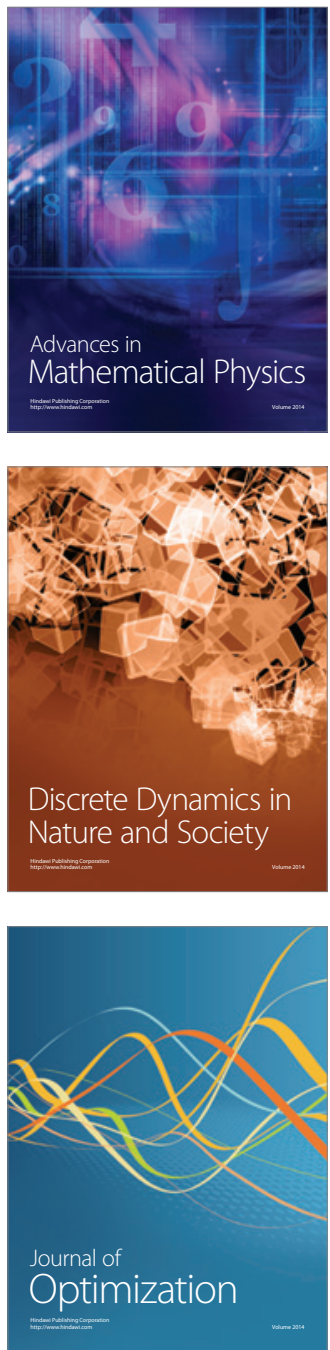